\title{
Contiguous Disease
}

National Cancer Institute

\section{Source}

National Cancer Institute. Contiguous Disease. NCI Thesaurus. Code C39767.

A term that usually refers to the clinical course of lymphomas and indicates that the cancerous lymph nodes are next to each other. 